\title{
Digital Chest Tomosynthesis
}

National Cancer Institute

\section{Source}

National Cancer Institute. Digital Chest Tomosynthesis. NCI Thesaurus. Code C126697.

Digital tomosynthesis used to image structures in the thoracic cavity. 